\title{
A Study of Emoticon Use in Instant Messaging from Smartphone
}

\author{
Tae Woong Park ${ }^{1}$, Si-Jung Kim² ${ }^{2}$, and Gene Lee ${ }^{1}$ \\ ${ }^{1}$ University of Central Florida, Industrial Engineering and Management Systems, \\ Orlando, Florida, USA \\ taewoong.park@knights.ucf.edu, glee@ucf.edu \\ ${ }^{2}$ University of Central Florida, School of Visuial Arts and Design - Digital Media, \\ Orlando, Florida, USA \\ sjkim@mail.ucf.edu
}

\begin{abstract}
Instant messaging (IM) has become one of the most popular modes of instantaneous electronic communication mediums throughout the world for users. A unique feature of IM on the smartphone is its choice of text-based, graphical or animated emoticons that express emotion and intentions. Many studies have been conducted on computer mediated communication (CMC), IM and emoticons, but little is known specifically about the study of emoticons in IM on the smartphone. The goal of this study was to understand how emoticons are used in IM on the smartphone and to investigate the inconsistent results of previous research. Thus, this study explored the frequency and variety of emoticon usage as well as the user traits based on emoticon types, relationships between users, availability of computer keyboards, and user emotional states in IM on the smartphone. The corresponding suggestions provided by this study would help to increase the understanding of emoticon usage and the designing of future emoticons in IM on the smartphone.
\end{abstract}

Keywords: Emoticon, Emotion, Instant Messaging (IM), Smartphone, Computer mediated communication (CMC).

\section{$1 \quad$ Introduction}

Traditional social interaction is face-to-face but today it is no longer the primary way for communicating with one another as there are many other alternative computer mediated communication (CMC) methods such as e-mail, text message and instant messaging (IM). A text-based CMC has a long history of sending text messages over networks and has been available since the 1960s. In the last decade, e-mail has been the prevalent communication tool of internet users [1]. Text messaging, such as IM, has been the prominent way of communication throughout the world [2]. IM is a popular mode of instantaneous electronic communication as well. Nardi and Whittaker suggested that IM is used mainly for four functions: (1) quick questions and clarifications, (2) organization and scheduling of work tasks, (3) organization of spontaneous social meetings, and (4) keeping in touch with friends and family [4]. Currently, there 
is IM software available via the internet such as Kakao Talk, AOL Instant Messenger, Google Talk, MSN Messenger, Yahoo Messenger and Facebook. As CMC replaces some face-to-face interaction, text-based communication methods, such as IM, are limited in expression and prone to misunderstandings. Face-to-face conversation depends on back channels such as the tone of voice, body language and interpretation of the listener's expressions, but text-based communication loses these back channels completely [5]. The back channel in face-to-face communication may serve different functions: (1) providing information; (2) regulating interaction; and (3) expressing intimacy [6]. Thus, CMC, such as IM, lacks the conventional non-verbal dimensions of human communication such as facial expressions, gestures, body positions, personal distance, vocal variety, and eye contact [7].

Consequently, finding ways to enrich the text-based communication is important. CMC users have found ways to increase the richness of CMC. The emoticon was created for this purpose. The use of the emoticons was well documented in a variety of sources in the CMC literature. There were a number of slightly varying definitions regarding emoticon. Antonijevic defined emoticons as emotional icons and visual representations of facial expressions used in CMC in order to represent the mood and/or emotion of the user [8]. The visual cues, such as emoticons, have been remarked as being the primary way to express emotion in CMC and are ways to replace non-verbal communications when not face-to-face $[9,10]$. Text messages without emoticons result in incorrect interpretation of the nature of the message and the senders' emotion or attitude [11]. Another study showed that children perceived higher degrees of social presence and greater intrinsic motivations when they were exposed to animated emoticons compared to when they were exposed to traditional emoticons [12]. The first person documented to have used the emoticons :-) (happy) and :-( (sad) was Scott E. Fahlman using a series of symbols to indicate mood in the body of an e-mail to prevent miscommunications from happening on September 19th, 1982. Recently, graphical emoticons have been introduced in IM and email and examples are shown in Table 1[1].

Table 1. Text-based and Graphical emoticon examples [1]

\begin{tabular}{lll}
\hline \multirow{2}{*}{ Emotion } & \multicolumn{2}{c}{ Emoticon } \\
\cline { 2 - 3 } & Text based & Graphical \\
\hline Happy & $:-)$ & \\
\hline Sad & $:-($ & \\
\hline Cry & $; \sim($ & \\
\hline Laughing & $:-\mathrm{D}$ & \\
\hline Wink & $;-)$ &
\end{tabular}

Since the introduction and consequential booming of the smartphone, smartphone users frequently send and receive instant messages with emoticons via IM in one's everyday life. A unique feature of IM on the smartphone is allowing usage of text based emoticons as well as graphical and animated emoticons. Smartphone users convey meaning using emoticons to represent individual identity, presence, awareness and feelings. 
Although many studies have been conducted on general CMC, IM and emoticons, most studies have focused on emoticon use in order to better understand the effect of emoticons on interpreting messages, but little is known specifically about the study of emoticons in IM on the Smartphone. The smartphone is being adopted at an exceptional rapidity, but little is known today about how people use these devices. In 2009, smartphone diffusion in the United States was $25 \%$ and $14 \%$ of worldwide mobile phone shipments were smartphones. In 2011, smartphone sales already exceeded desktop PCs. Falaki and Mahajan investigated the view of what users use their smartphones for and the communication dominates as a result of this study [13]. In conclusion, the use of IM on the smartphone is likely to increase at a rapid rate.

\section{Purpose of Study}

\subsection{Research Questions}

This study is an exploratory study with the survey research questions. First of all, this study is intended to identify the general characteristics of emoticon use. It was expected that the trait of emoticon use in IM on the smartphone would show a different tendency than in IM on the computer.

There are many emoticons used in IM, but emoticon users do not use all emoticons. Danet showed that the best known ones are a :-) (smile), ;-) (wink), and :-((frown) [14]. Garrison and Remley investigated that use of three emoticons, :-) (happy/smile), :-P ( playfulness) and ;-) (wink) run to $73 \%$ and the :-) (happy/smile) was the most used emoticon (35\%) in his data set [3]. Tossell and Kortum showed that the top three emoticons are :) (happy), : ( (sad), and :D (very happy) and these made up $70 \%$ of the total amount of emoticons sent across all participants [2]. In this line of reasoning, this study investigated what specific style of emoticons is the most commonly used in IM on the smartphone (Question 1).

With the introduction of various media in CMC, graphical emoticons, as well as text-based emoticons, became very popular. Furthermore, as animated emoticons became more widely supported, countless animated emoticons were created and continue to be created today. This study examined whether users prefer text-based :-), graphical $\because$ or animated emoticons $\because$ (actually moving) when users send emoticons with instant messages on their smartphones (Question 2).

CMC users often incorporate emoticons as visual cues to augment the meaning of textual electronic messages [15]. It appears that users of CMC attempt to compensate for the lack of the traditional nonverbal cues by the usage of emoticons [16]. Though emoticons are defined as a means to express one's emotion, some people would like to conceal emotion when in a negative emotional state or to make a warm and bright atmosphere in an IM. To attain this intention, one sends a text message with a :-) (happy emoticon) [22]. In this line of reasoning, this study examined the main intention or reasoning for the use of emoticons in IM on the smartphone (Question 3). 
In the Tossell's study, the authors collected real communications data from individuals' smartphones over a 6-month period. In general, SMS messages were not used very much overall, with only $4 \%$ of all messages containing at least one emoticon [2]. Ling analyzed 882 messages using phone interviews to collect data and showed that only $6 \%$ of these messages contained emoticons [17]. On the contrary, in other survey-based research, Qiao showed that $88 \%$ of a Chinese sample used emoticons. Findings also showed that Chinese highly used emoticons in SMS for humor and as a substitute for non-verbal communication [18]. Because of these previous inconsistent results, this study examined how many emoticons were included in IM on the smartphone (Question 4).

Thus, the primary research questions related to emoticon use were presented as follows: (1) What specific styles of emoticons do most people like? (2) Out of the three types of emoticons (text-based, graphical and animated emoticons) which do most people prefer? (3) What is the main reason or intention most people send emoticons? (4) How many emoticons do most people send in an instant message on the smartphone?

\subsection{Hypotheses}

Next, this research was attracted to investigating existing research of emoticon use because other studies have had inconsistent results. On the basis of the results of our prior research questions', we analyzed the aspect or attitude of emoticon users, considering various factors related to the use of IM from a user's smartphone with specific research hypotheses. In particular, how the availability of a computer keyboard, gender, user's emotional state, and the relationship of those texting affects the frequency of emoticon use.

The computer keyboard is still one of the most commonly used input devices today. It allows rapid entry of text by experienced users. It is easy to enter textual data using the computer keyboard when one uses instant messenger on the computer. Today, it is commonplace to see users who are tapping their fingers on the small virtual keyboards of their smartphones but people feel an inconvenience or irritation when one uses instant messenger on the smartphone. In addition, smartphone users are often experiencing a difficulty in typing characters exactly on minute keys due to their thick thumbs [19]. In this line of reasoning, do people send emoticons in IM from smartphones in the same manner one sends emoticons with a computer keyboard? Individuals at least feel the need to convey some of their emotions or points with emoticons rather than long text. It is faster to use emoticons than words to convey certain expressions based on the result of question 3. Therefore, this study expected that people would use more emoticons on the smartphone than on the desktop or laptop with an established standard keyboard (Hypothesis 1).

There were a few studies regarding gender difference. There are other studies that have had inconsistent results. In particular, there was disagreement within the research about the effect of gender on the frequency of emoticon usage. In one study, across all mediated communication methods, there were no statistical gender differences [20]. In another study, Derks and Bos [21] also showed that females use more 
non-verbal communication in face-to-face encounters. In the same manner, males sent less emoticons in text messages compared to females [2]. Luor and $\mathrm{Wu}$ found that the intention of using emoticons was not statistically significant in terms of gender, but data suggested that females used more emoticons [22]. However, these studies only considered the gender of the sender and not the relationship with the gender of the receiver. This study investigated how often males and females use emoticons in instant messages with both genders. In terms of gender, females will send more emoticons in instant messages to males than to females and males will send more emoticons in instant messages to females than to males (Hypothesis 2).

An interaction was found between valence and the kind of context; in negative or task-oriented contexts people used the least emoticons [20]. When chatting in IM on the smartphone, it was interesting to know whether people would send more emoticons in a positive emotional state than in a negative emotional state (Hypothesis 3).

The frequency of nonverbal emotions in face-to-face interaction increases when the interacting partner was considered to be a friend [23]. Text messages are mostly private communications with friends [24]. In this line of reasoning, when chatting privately as well as officially in IM, it was interesting to know whether horizontal relationships (equal-equal, e.g., friends) would result in greater emoticon usage than vertical relationships (superior-inferior, e.g., supervisor) (Hypothesis 4).

To sum up, this study tested the following four research hypotheses related to emoticon use: (1) There will be more emoticon usage on the smartphone than on the computer. (2) Users will send more emoticons in instant messages to users of the opposite sex, than to the same sex. (3) People will send more emoticons in good emotional state than in bad emotional state. (4) Horizontal relationships will result in greater emoticon usage than vertical relationships.

\section{$3 \quad$ Research Methods}

\subsection{Participants}

A total of 53 people participated in this survey. This study investigated the feature of emoticon use among people who use IM on the smartphone. The participants consisted of 29 males $(54.7 \%)$ and 24 females $(45.3 \%)$. There are four participants in the 10-20 years age range, 48 participants in the 30-40 range, and one participant in the over 50 range. Over 48 of the participants $(90.6 \%)$ were $30-40$ years old.

\subsection{Independent and Dependent Variables}

For four primary research questionnaires, this study applied descriptive statistics. On the other hand, for testing the research hypothesis, the Mann-Whitney U test was conducted to compare user's attitudes with respect to several hypotheses and a single Likert scale question was written as 5 point scale ranging from "Strongly Disagree" on one end to "Strongly Agree" on the other and "Neither Agree nor Disagree" in the 
middle. This is a non-parametric test, and is therefore well suited to our Likert scale data because we cannot presume that the population of the collected data fits a normal distribution. Specifically, four independent variables were considered to test the research hypotheses: availability of the computer keyboard (a computer keyboard and a smartphone keyboard), gender (male and female), emotional states (positive and negative) and human relationship (vertical and horizontal). Dependent variables of this study were user's attitudes about frequency of emoticon use.

\subsection{Procedures and Questionnaire}

A web survey research was conducted to see how emoticons are used in IM on the smartphone. After designing the survey research, it was sent to participants via Internet and tracked during two weeks in October 2012. A set of questionnaires were developed that has three sections: basic information of participants, primary questionnaire and research hypotheses about emoticon use. Participants received a questionnaire, which he/she filled out individually. For research questions, each of these was measured by multiple items in the questionnaire. The respondents were permitted to react by given a text message, by selecting the frequency and by picking emoticons from a list of thirty-seven emoticons. For research hypotheses, the respondents were asked to indicate their level of agreement with a given statement by way of an ordinal scale. This survey took approximately 15 minutes of his/her time.

\section{$4 \quad$ Results and Discussion}

\subsection{Results}

The results of four primary research questions related to emoticon use were presented as follows: First, there are many emoticons used in IM, but emoticon users did not use all emoticons. Participants showed a great dependency on just five of all possible 37 emoticons to be used and implemented, and utilized only 14 different emoticons in total. For users' first choice of emoticons, the five emoticons used mostly by the participants were chuckle $(\Theta), 32.1 \%)$, smile $(\ominus, 20.8 \%)$, beam $(\Theta, 15.1 \%)$, wink ( $\bullet, 7.5 \%)$, and crushed $(\because, 5.7 \%)$. As the findings show, emoticon styles that most users preferred were similar to a smiling face in the survey.

Second, although graphical emoticons as well as animated emoticons became very popular, out of the three types of emoticons (text-based, graphical and animated emoticons), text-based emoticons were more preferred $(39.6 \%)$ compared to the other two, graphical $(34.0 \%)$ and animated emoticons $(26.4 \%)$.

Third, we have identified the main reason and intention most people send emoticons in IM on the smartphone. They were as follows: (1) Emoticons can help the receiver understand more accurately and clearly $(35.0 \%)$, (2) To make a warm and bright atmosphere in IM (22.5\%), (3) Using emotions is easier than to write text 
messages in IM (18.8\%), (4) It is fun to use emoticons in IM (15.0\%), (5) Using emoticons is popular now in IM $(6.2 \%)$ and (6) The other response $(2.5 \%)$. These results mean that emoticon use was considerably correlated to enforcing richness of information exchange. To make the conversation smooth and bright is not the first reason.

Lastly, when people IM from start to finish on a smartphone, the frequency of emoticons were as follows: Most participants tend to use at least one emoticon when they are texting. The frequency of emoticon use analysis showed that only three participants $(5.7 \%)$ are not using emoticons when texting. The other distribution of the emotion use is that one emoticon is used $(37.7 \%)$, two to three emoticons are used $(28.3 \%)$, four to five emoticons are used (3.8\%) and over six emoticons are used $(24.5 \%)$.

In the second place, this study tested the research hypotheses related to emoticon use: First, will emoticons be used more on the smartphones rather than on the non-mobile devices such as laptop and desktop? There was a difference between the frequency of emoticons on the smartphone and on the computer. There was more emoticon use on the smartphone than on the computer $(\mathrm{U}=389.5, \mathrm{p}<.01$, one-tailed $)$ Thus, hypothesis 1 was confirmed.

Second, there were statistical gender differences. Users sent more emoticons in IM to users of the opposite sex, than to the same sex (same sex Mdn = 1, opposite sex $M d n=2, U=2207.5, p<.01$, one-tailed). This means that hypothesis 2 was confirmed.

Third, interestingly, there was a difference between the frequency of emoticon use in good emotional states and the frequency of emoticon use in bad emotional states. The participants tend to use emoticons in their good emotional states more than in their bad emotional states $(\mathrm{U}=709.5, \mathrm{p}<.01$, one-tailed). Hypothesis 3 was confirmed.

Lastly, there was a difference between the frequency of emoticons on the horizontal relationships between friends and on the vertical relationships between supervisor and worker. People send more emoticons on the horizontal relationships than on the vertical relationships $(\mathrm{U}=765.5, \mathrm{p}<.01$, one-tailed). Hypothesis 4 was confirmed.

\subsection{Discussion}

The present study about emoticon use showed interesting results. Participants showed a great dependency on just five emoticons. Most users preferred emoticons that were similar to a smiling face. This implies that participants would like to express their positive feelings with others rather than expressing their negative emotional feelings. It identified that people prefer more emoticons to represent a smiling face than emoticons to represent a scowling face (Question 1). This result corresponds with research from Lee and Wagner which showed that people displayed more emotions in positive contexts than in negative contexts [25]. Although, IM on the smartphone is more accessible to express negative emotions, people are reluctant to use negative emoticons even in this kind of electric communication. On the other hand, people who do not use 
emoticons in IM replied that: (1) Sending emoticons in IM from a smartphone is annoying. (2) Using emoticons in IM interrupts the communication. (3) Emoticons make chatting conversations less serious.

The text-based emoticons were preferred over graphical and animated emoticons (Question 2). The main reason was that it is easy to type the emoticons directly on the smartphone keyboard without needing to find particular emoticons. In other words, emoticon users wanted the speed to type emoticons and convenient access to emoticons. However, people who prefer graphical emoticons presented different opinions and emotions or feelings that he/she can express more exactly and diversely than through text-based emoticons. Also, he/she replied that animated emoticons are more actively appealing but not professional. On the other hand, people who prefer animated emoticons gave his/her opinions that they can communicate emotions and feelings more strongly by them than through graphical or text-based emoticons.

The results were analyzed regarding the main reason and intention most people send emoticons. Emoticon use was considerably correlated to enforcing richness of information exchange (Question 3). Senders want receivers to understand instant messages well, so he/she sends emoticons to prevent miscommunication. Next, some emoticon users sent emoticons to induce a warm environment before stating IM.

Tossel and Kortum showed that over 158,098 text messages were sent and received by his 21 participants and only $4 \%$ contained emoticons [2]. In contrast to his findings, this study showed that the frequency of emoticons use was varied when people IM from start to finish on a smartphone. Particularly, this study showed that participants send messages including zero emoticons $(5.7 \%)$. The difference of results can be outcome of cultural or nationality differences between the two countries. It can be concluded that people who used three or fewer emoticons have an intention such as an expression of emotion, richness of communication and a bright warm mood. Over four emoticons users showed that emoticon use could become habitual and less conscious in IM.

As the result of the research hypotheses test, hypothesis 1 was confirmed. People use more emoticons on the smartphone than on the desktop computer or laptop. Speed and accuracy of communication are important considerations in the design of interactive systems. Although the layout of the letters on a smartphone keyboard is similar to a computer keyboard, the arrangement of a smartphone's keys is not optimal for typing. This resulted in more text messages displaying several emoticons because people have not become accustomed to virtual keyboards of smartphones.

Hypothesis 2 was confirmed as well. Users sent more emoticons in IM on the smartphone to users of the opposite sex, than to the same sex. This result was found from the receiver's sex. Some studies showed that females are more intensive emoticon users than males. Luor and Wu already showed that females had a higher frequency of using emoticons to express their emotions when using IM compared with males [22], but the above studies need to be analyzed in terms of the receiver's sex.

Hypothesis 3 was confirmed as well. People sent more emoticons in good emotional states than in bad emotional states. This result supports the finding that we described 
earlier that most participants preferred to use positive emoticons rather than negative emoticons. This study concluded that emoticons are not used as frequently in negative moods as most users preferred emoticons representing smiling faces. Clearly, the emoticons that are used to express negative emotions are not frequent in a negative situation.

Hypothesis 4 was confirmed as well. People send more emoticons on the horizontal relationships (equal-equal) than on the vertical relationships (superior-inferior). It is more appropriate to show one's emotions and feelings towards friends or colleagues than towards supervisors or seniors. The expression of emotions in IM from a smartphone, by use of emoticons, is similar to the expression of emotions in face-to-face communication. Most people want to be very sincere and professional to his/her supervisors or elders. Although, emoticons are not actual non-verbal behavior, this fact was showed in electronic communication.

\section{Conclusion and Future Work}

This study intended to uncover the user's trait associated with emoticon use in IM on the smartphone. The overall conclusion of this study is that traits of emoticon users in IM on the smartphone are similar to in face-to-face communication. The primary of emoticon use was to help them communicate accurately. Emoticons are being used to represent their emotions or feeling as well as to make their conversations smooth and represented in positive mood. In addition, emoticons are being used more between the different genders rather than the same gender. Also, emotions are being used more among the same horizontal relationships rather than among the vertical relationships. It is our belief that the characteristics of emoticon use that we found from this study would contribute to the understanding of emoticon use in IM on the smartphone and further that they would serve as a base for developing and improving the next levels of emoticon. The next step of this research is to conduct another user study with a diverse group of participants that will provide more characteristics of emoticon use.

\section{References}

1. Huang, A.H., Yen, D.C., Zhang, X.: Exploring the potential effects of emoticons. Information \& Management 45(7), 466-473 (2008)

2. Tossell, C.C., et al.: A longitudinal study of emoticon use in text messaging from smartphones. Computers in Human Behavior 28(2), 659-663 (2012)

3. Garrison, A., et al.: Conventional Faces: Emoticons in Instant Messaging Discourse. Computers and Composition 28(2), 112-125 (2011)

4. Nardi, B.A., Whittaker, S., Bradner, E.: Interaction and outeraction: instant messaging in action. In: Proceedings of the 2000 ACM Conference on Computer Supported Cooperative Work. ACM (2000)

5. Dix, A., et al.: Human Computer Interaction, 3rd edn. (2004)

6. Harrison, R.P.: Nonverbal Communication. Human Communication As a Field of Study: Selected Contemporary Views, 113 (1989)

7. Rogers, E.M., Allbritton, M.M.: Interaction Communication Technologies in Business Organizations. The Journal of Business Communication 32(2), 177-195 (1995) 
8. Antonijevic, S.: Expressing Emotions Online: An Analysis of Visual Aspects of Emoticons. In: EEO Conference Papers: International Communication Association 2005 Annual Meeting, New York (2005)

9. Walther, J.B., D'Addario, K.P.: The Impacts of Emoticons on Message Interpretation in Computer-Mediated Communication. Social Science Computer Review 19(3), 324-347 (2001)

10. Riva, G.: The Sociocognitive Psychology of Computer-Mediated Communication; The Present and Future of Technology-Based Interactions. Cyberpsyc Hology \& Behavior 5, 581-598 (2002)

11. Lo, S.K.: The nonverbal communication functions of emoticons in computer-mediated communication. CyberPsychology \& Behavior 11(5), 595-597 (2008)

12. Tung, F.-W., Deng, Y.-S.: Increasing social presence of social actors in e-learning environments: Effects of dynamic and static emoticons on children. Displays 28(4-5), 174-180 (2007)

13. Falaki, H., et al.: Diversity in smartphone usage. In: Proceedings of the 8th International Conference on Mobile Systems, Applications, and Services. ACM (2010)

14. Danet, B., Ruedenberg-Wright, L., Rosenbaum-Tamari, Y.: Smoking dope at a virtual party: Writing, play and performance on Internet Relay Chat. Network and Netplay: Virtual Groups on the Internet (1997)

15. Rezabek, L.L., Cochenour, J.J.: Visual Cues in Computer-Mediated Communication: Supplementing Text with Emoticons. Journal of Visual Uteracy 18(2), 201-215 (1998)

16. Krohn, F.B.: A generational approach to using emoticons as nonverbal communication. Journal of Technical Writing and Communication 34(4), 321-328 (2004)

17. Ling, R.: Mobile communications vis-à-vis teen emancipation, peer group integration and deviance. The Inside Text, 175-193 (2005)

18. Qiao, Q.: The adoption and use of SMS among Chinese teenagers (2010)

19. Kwon, T., Na, S., Park, S.-H.: Drag-and-Type: A new method for typing with virtual keyboards on small touchscreens. In: 2013 IEEE International Conference on Consumer Electronics (ICCE). IEEE (2013)

20. Derks, D., Bos, A.E.R., Grumbkow, J.V.: Emoticons and social interaction on the Internet: the importance of social context. Computers in Human Behavior 23(1), 842-849 (2007)

21. Derks, D., Bos, A.E.R., Von Grumbkow, J.: Emoticons and online message interpretation. Social Science Computer Review 26(3), 379-388 (2008)

22. Luor, T., et al.: The effect of emoticons in simplex and complex task-oriented communication: An empirical study of instant messaging. Computers in Human Behavior 26(5), 889-895 (2010)

23. Wagner, H., Lee, V.: 10. Facial Behavior Alone and in the Presence of Others. The social context of nonverbal behavior, p. 262 (1999)

24. Häkkilä, J., Chatfield, C.: 'It's like if you opened someone else's letter': user perceived privacy and social practices with SMS communication. In: Proceedings of the 7th International Conference on Human Computer Interaction With Mobile Devices \& Services. ACM (2005)

25. Lee, V., Wagner, H.: The effect of social presence on the facial and verbal expression of emotion and the interrelationships among emotion components. Journal of Nonverbal Behavior 26(1), 3-25 (2002) 


\section{Appendix}

Table 2. Graphical emoticons of Kakao Talk

\begin{tabular}{|c|c|c|c|}
\hline Emotion & Meaning & Emoticon & Meaning \\
\hline$(-8)$ & Sulk & 3 & Cute \\
\hline 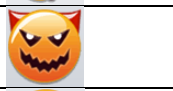 & Devil & (2:0) & Bubble \\
\hline का & Puke & $\infty$ & Cool \\
\hline 0 & Embarrassed & 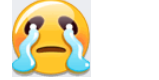 & Cry \\
\hline 60 & Blush & 20 & Pish \\
\hline 0 & Surprised & 80 & Alien \\
\hline-3 & KiKi & -0 & Sleepy \\
\hline$\theta$ & Wink & ( ) & Satisfied \\
\hline$=$ & Frustrated & 0 & Confused \\
\hline 5 & Worried & 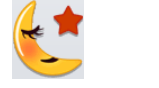 & Sleeping \\
\hline$\Xi$ & Relieved & 0 & Crushed \\
\hline in & Grumpy & 25 & Sneer \\
\hline 0 & Angry & 8 & Alien-girl \\
\hline 89 & Tipsy & 10 & Hesitant \\
\hline 60 & Oh, my goodness & 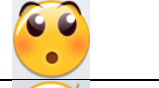 & Curious \\
\hline 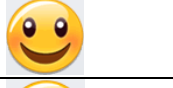 & Smile & $\times x$ & Sick \\
\hline 80 & Hurray & 25 & Tongue \\
\hline$\because$ & Scared & 6 & Beam \\
\hline-2 & happy & & \\
\hline
\end{tabular}

